\title{
Channel Probing in the Uplink of OFDM-based Wireless Systems
}

\author{
Seung-Hwan Lee and Yong-Hwan Lee \\ School of Electrical Engineering and INMC \\ Seoul National University \\ Kwanak P. O. Box 34, Seoul 151-600 Korea \\ E-mail: leesh80@ttl.snu.ac.kr, ylee@snu.ac.kr
}

\begin{abstract}
In this paper, we consider a new channel probing scheme for opportunistic scheduling in the uplink of orthogonal frequency division multiplexing (OFDM)-based wireless systems. To reduce signaling overhead for the probing, we consider a twostep channel probing process; called pre-probing and mainprobing. Good subchannels are first estimated through the preprobing process using a probing signal transmitted through subcarriers spread out the whole bandwidth. Then, by sending a main-probing signal over only good subchannels, the proposed scheme can significantly reduce the probing signaling overhead without noticeable performance degradation compared to the full probing scheme. Finally, the performance of the proposed scheme is verified by computer simulation.
\end{abstract}

\section{INTRODUCTION}

Orthogonal frequency division multiplexing (OFDM) has been considered as one of the most promising transmission techniques for broadband wireless access. OFDM systems can increase the spectral efficiency by exploiting so-called multiuser diversity (MUD) gain [1], [2]. Recently, the use of opportunistic transmission has been applied to the uplink as well as the downlink [3], [4]. OFDM signals are often transmitted through subchannels each of which comprises several subcarriers.

To employ opportunistic transmission in the uplink, the base station (BS) needs to know the channel condition of mobile stations (MSs). The BS can estimate the channel condition using a probing signal transmitted from the MS. For example, IEEE 802.16e uses a probing signal pre-determined during the initial access or handshaking process [4]. However, it may require heavy signaling burden to provide desired performance since the probing signal is transmitted over contiguous subchannels without considering the channel characteristics of MS. As a consequence, it may be quite inefficient in the presence of high correlation between the adjacent subchannels.

In this paper, we propose a new channel probing scheme comprising two steps, pre-probing and main-probing.

First, it is desirable to find out subchannels in good channel condition. To this end, the MS transmits a known pre-probing signal which covers up the whole frequency band. To minimize the signaling overhead for the pre-probing, the pre-probing signal is transmitted over every several subcarriers. Once the MS knows subchannels in good channel condition, it transmits another known signal, called main-probing signal, only through good subchannels. By exploiting the two-step approach, each MS can utilize subchannels in good channel quality for the signal transmission, without increasing the probing signaling overhead. It is shown that as the number of MS increases, the proposed probing scheme can provide the almost same performance as full probing scheme that use a probing signal that occupies all subcarriers.

The rest of paper is organized as follows. Section II describes the system model in consideration. The proposed two-step channel probing scheme is described in Section III and verified by computer simulation in Section IV. Finally, conclusions are summarized in Section V.

\section{System MODELiNG}

Consider an uplink OFDM system with $K$ active users, where the whole bandwidth is divided into $M$ subchannels comprising $N$ subcarriers, resulting in total $M N$ subcarriers. Let $X_{k}(n, t)$ be the data symbol of MS $k$ for the $n$-th subcarrier at time $t$. Assuming that $X_{k}(n, t)$ is transmitted over an additive noise channel $H_{k}(n, t)$, we can represent the received signal $Y_{k}(n, t)$ as

$$
Y_{k}(n, t)=H_{k}(n, t) X_{k}(n, t)+N(n, t)
$$

where $H_{k}(n, t)$ is assumed to be a zero mean complex Gaussian random variable with unit variance and $N(n, t)$ represents additive noise term. Let $\tilde{H}_{k}(m, t)$ be the averaged channel gain of the $m$-th subchannel of MS $k$ at time $t$. Then, it can be obtained by averaging the channel gain of $N$ subcarriers belonging to the subchannel as

$$
\tilde{H}_{k}(m, t)=\frac{1}{N} \sum_{n=0}^{N-1} H_{k}(m N+n, t) .
$$

Assuming that all the MSs have the same average signal to noise ratio (SNR) equal to $\gamma_{o}$, the corresponding instantaneous SNR of MS $k$ for the $m$-th subchannel at time $t$ can be represented as

$$
\gamma_{k}(m, t)=\left|\tilde{H}_{k}(m, t)\right|^{2} \gamma_{o}
$$




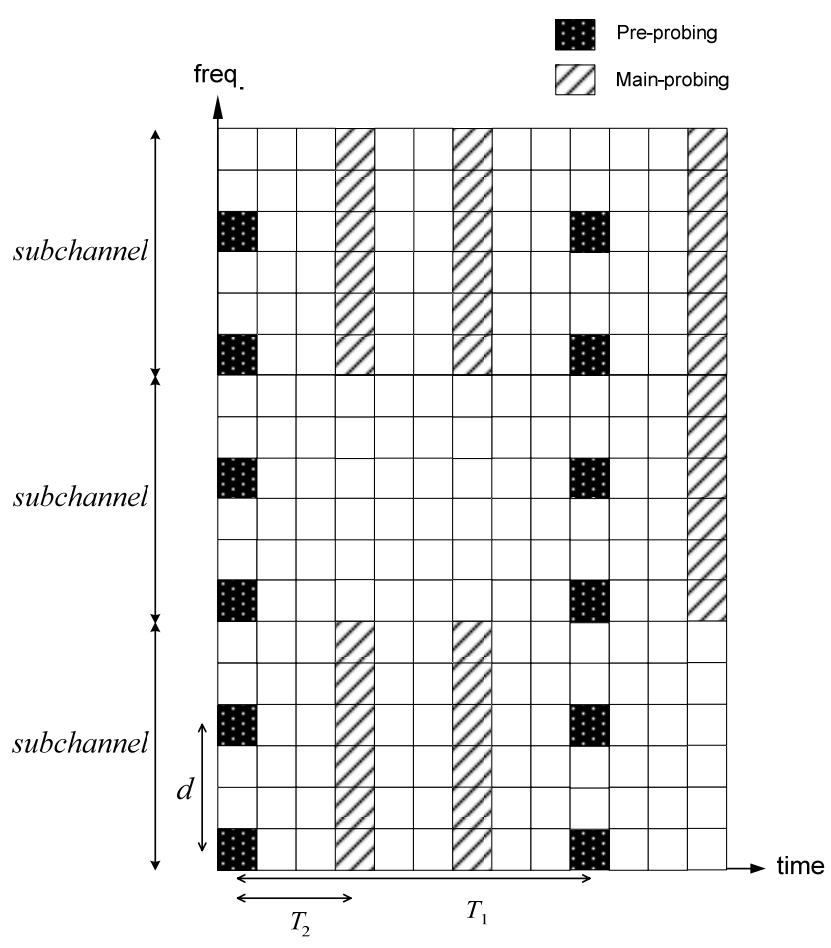

Fig. 1. Processing concept of the proposed probing scheme.

Assuming that the channel characteristics are invariant over each subchannel, the probability density function (pdf) and the cumulative distribution function (cdf) of $\gamma_{k}(m, t)$ can respectively be given by

$$
\begin{gathered}
f_{\gamma}\left(\gamma_{k}\right)=\frac{1}{\gamma_{o}} \exp \left(-\frac{\gamma_{k}}{\gamma_{o}}\right), \quad \gamma_{k} \geq 0, \\
F\left(\gamma_{k}\right)=\int_{0}^{\gamma_{k}} f_{\gamma}(x) d x .
\end{gathered}
$$

\section{Proposed Probing SCHEME}

We consider the probing comprising two steps, pre-probing and main-probing, which are performed every $T_{1}$ and $T_{2}\left(\leq T_{1}\right)$ seconds respectively as shown in Fig. 1 . Here, the probing period $T_{1}$ and $T_{2}$ can be adjusted according to the channel condition (e.g., the user mobility) to minimize the signaling overhead for the channel probing. During the preprobing period, each MS transmits a pre-probing signal over the whole frequency band to determine subchannels in good channel condition. Once each MS knows subchannels in good condition, it transmits a main-probing signal only over good subchannels to accurately estimate the channel condition. Since MSs can consider the use of the same subchannels simultaneously, the scheduler can additionally achieve a multiuser diversity (MUD) gain.

In the first step, MS $k$ transmits pre-probing signal $P_{k}^{p}(n, t)$ over every $d(\geq K)$ subcarrier as illustrated in Fig. 1, i.e.,

$$
P_{k}^{p}(d l+k, t)=c^{p}(l, t)
$$

where $c^{p}(l, t)$ denotes the $l$-th signal for the pre-probing at time $t$ and the superscript $p$ indicates the pre-probing step. Note that the orthogonality among the MSs can be maintained by sending pre-probing signals through a set of non-overlapped subcarriers. By probing the channel condition through the whole frequency band, the BS determines a set of subchannels $S_{k}$ having good channel condition (i.e., the SNR larger than threshold $\alpha_{k}$ ) for each MS as

$$
S_{k}=\left\{\hat{m}_{k} \mid \gamma_{k}\left(\hat{m}_{k}, t\right) \geq \alpha_{k}\right\} .
$$

Here, $\alpha_{k}$ can be determined by considering the estimated SNR $\gamma_{k}(m, t)$ of the $m$-th subchannel at time $t$.

In the second step, MS $k$ transmits the main-probing signal $P_{k}^{m}(n, t)$ through subchannels in $S_{k}$ determined by (8) as

$$
P_{k}^{m}\left(\hat{m}_{k} N+l, t\right)=c^{m}(l, t) \exp \left(\frac{-j 2 \pi n k}{\mu}\right), \quad \hat{m}_{k} \in S_{k}
$$

where $c^{m}(l, t)$ is the $l$-th pilot symbol for the main-probing at time $t, \mu$ is the phase shift index [5] and the superscript $m$ indicates the main-probing step. Since a phase shift in the frequency domain results in a delay in the time domain, the orthogonality among the MSs can be maintained by making the amount of phase shift $\mu$ be equal to the number of MSs that simultaneously transmit the main-probing signal over the same subchannel [3], [5], [7]. Through the main-probing process, the BS can not only accurately estimate the channel condition to determine the data rate for the subchannel, but also achieve the MUD gain by choosing a MS in the best channel condition.

In the proposed scheme, the BS can determine the preprobing interval $T_{1}$ and the main-probing interval $T_{2}$ for each MS according to the channel correlation in the time domain. If $T_{1}$ is set to a large value, the system throughput can be reduced since the outdated channel gain of good subchannel can easily be worse. On the other hand, if $T_{1}$ is set to a too small value, it may also result in a reduction of the system throughput because the pre-probing signal requires a large amount of resource. Similarly, as $T_{2}$ increases, the data rate previously determined may not be appropriate to the current channel condition and thus reducing the system throughput. But as $T_{2}$ decreases, the overhead for the mainprobing may reduce the overall system throughput. Thus, it is required to properly determine the value of $T_{1}$ and $T_{2}$ considering both the channel characteristics and signaling overhead.

Let $R_{k}(\Delta t)$ be the correlation between the channels at time $t$ and $t+\Delta t$ defined as

$$
R_{k}(\Delta t) \cong E\left\{H_{k}(n, t) H_{k}^{*}(n, t+\Delta t)\right\}
$$


TABLE I

SIMULATION PARAMETERS

\begin{tabular}{cc}
\hline \hline PARAMETERS & Values \\
\hline Number of subcarriers per subchannel $(N)$ & 36 \\
Number of subchannels $(M)$ & 24 \\
Average SNR $\left(\gamma_{o}\right)$ & $10 \mathrm{~dB}$ \\
Doppler spread $\left(f_{D}\right)$ & $21.3 \mathrm{~Hz}$ \\
rms delay spread $\left(\tau_{r m s}\right)$ & $2300 \mathrm{~ns}$ \\
Pre-probing interval $\left(T_{1}\right)$ & $25 \mathrm{~ms} \quad(R(\Delta t) \approx 0.1)$ \\
Main-probing interval $\left(T_{2}\right)$ & 5 ms $\quad(R(\Delta t) \approx 0.85)$ \\
HARQ & Chase combine with max \\
& retransmission of 3 \\
\hline \hline
\end{tabular}

where the superscript $*$ denotes complex conjugate and $E\{X\}$ denotes the expectation of random variable $X$. Then, $T_{1}$ and $T_{2}$ can be determined as

$$
\begin{aligned}
& \hat{T_{1}}=\arg \max _{T_{1}}\left\{R_{k}\left(T_{1}\right) \leq \delta_{1}\right\}, \\
& \hat{T_{2}}=\arg \max _{T_{2}}\left\{R_{k}\left(T_{2}\right) \leq \delta_{2}\right\}
\end{aligned}
$$

where $\delta_{1}$ and $\delta_{2}$ respectively are threshold values to be optimized considering the trade-off between the channel probing performance and the signaling overhead for the probing. Note that the pre-probing and main-probing should be performed disjointly to preserve the orthogonality between the probing signals.

For performance analysis of the proposed probing scheme, assume that all the MSs use the same threshold, i.e., $\alpha_{k}=\alpha$ for $k=0, \ldots, K-1$. First, we consider the case where the channel is invariant over the probing period, i.e., we assume no capacity loss due to the outdated channel information. We set the SNR of subchannels having an SNR less than the threshold value (i.e., $\gamma_{k}(m, t)<\alpha$ ) to zero (i.e., $\gamma_{k}(m, t)=0$ ) to prevent resource allocation. In this case, the pdf and cdf of the SNR can be represented respectively as [8]

$$
\begin{gathered}
f_{\alpha}\left(\gamma_{k}\right)= \begin{cases}0, & 0 \leq \gamma_{k}<\alpha \\
f\left(\gamma_{k}\right), & \gamma_{k} \geq \alpha,\end{cases} \\
F_{\alpha}\left(\gamma_{k}\right)= \begin{cases}F(\alpha), & 0 \leq \gamma_{k}<\alpha \\
F\left(\gamma_{k}\right), & \gamma_{k} \geq \alpha .\end{cases}
\end{gathered}
$$

Assuming the scheduler chooses a MS having the largest SNR, the system capacity can be represented as [10]

$$
\begin{aligned}
& C_{\text {prop }}=\int_{0}^{\infty} \log _{2}\left(1+\gamma_{k}\right) K F_{\alpha}\left(\gamma_{k}\right)^{K-1} f_{\alpha}\left(\gamma_{k}\right) d \gamma_{k} \\
& =\int_{\alpha}^{\infty} \log _{2}\left(1+\gamma_{k}\right) K\left(1-\exp \left(-\frac{\gamma_{k}}{\gamma_{o}}\right)\right)^{K-1} \frac{1}{\gamma_{o}} \exp \left(-\frac{\gamma_{k}}{\gamma_{o}}\right) d \gamma_{k} .
\end{aligned}
$$

By using the binomial expansion [11], this can be rewritten as

$$
C_{\text {prop }}=\int_{\alpha}^{\infty} \log _{2}\left(1+\gamma_{k}\right) K \sum_{i=0}^{K-1}(-1)^{i}\left(\begin{array}{c}
K-1 \\
i
\end{array}\right) \frac{1}{\gamma_{o}} \exp \left(-\frac{(1+i) \gamma_{k}}{\gamma_{o}}\right) d \gamma_{k} .
$$

On the other hand, when probing signal is transmitted over the whole bandwidth, the system capacity can be represented as

$$
\begin{aligned}
& C_{\text {full }}=\int_{0}^{\infty} \log _{2}\left(1+\gamma_{k}\right) K F\left(\gamma_{k}\right)^{K-1} f\left(\gamma_{k}\right) d \gamma_{k} \\
& =\int_{0}^{\infty} \log _{2}\left(1+\gamma_{k}\right) K \sum_{i=0}^{K-1}(-1)^{i}\left(\begin{array}{c}
K-1 \\
i
\end{array}\right) \frac{1}{\gamma_{o}} \exp \left(-\frac{(1+i) \gamma_{k}}{\gamma_{o}}\right) d \gamma_{k} .
\end{aligned}
$$

Define $C_{\text {loss }}$ by the capacity loss due to the use of the proposed scheme when compared to the use of full SNR information as

$$
C_{\text {loss }}=C_{\text {full }}-C_{\text {prop }} \text {. }
$$

It can easily be shown that

$$
\begin{aligned}
& C_{\text {loss }}=\int_{\alpha}^{\infty} \log _{2}\left(1+\gamma_{k}\right) K F\left(\gamma_{k}\right)^{K-1} f\left(\gamma_{k}\right) d \gamma_{k} \\
& =\int_{\alpha}^{\infty} \log _{2}\left(1+\gamma_{k}\right) K \sum_{i=0}^{K-1}(-1)^{i}\left(\begin{array}{c}
K-1 \\
i
\end{array}\right) \frac{1}{\gamma_{o}} \exp \left(-\frac{(1+i) \gamma_{k}}{\gamma_{o}}\right) d \gamma_{k} .
\end{aligned}
$$

It can be seen that

$$
\lim _{K \rightarrow \infty} C_{\text {loss }}=0
$$

because $F\left(\gamma_{k}\right)^{K-1}$ is decreased to zero due to that $F\left(\gamma_{k}\right)<1$.

Next, consider the capacity loss due to the use of outdated channel information. The channel gain $H_{k}(n, t+\Delta t)$ of the $n$-th subchannel at time $t+\Delta t$ can be expressed in term of $H_{k}(n, t)$ as [9]

$$
H_{k}(n, t+\Delta t)=R_{k}(\Delta t) H_{k}(n, t)+\sqrt{1-\left|R_{k}(\Delta t)\right|^{2}} z_{k}(n, t)
$$

where $z_{k}(n, t)$ is a zero-mean complex Gaussian random variable with unit variance and independent of $H_{k}(n, t)$. Then, the expected SNR of the $m$-th subchannel at time $t+\Delta t$ can be represented as

$$
\begin{aligned}
& \gamma_{k}(m, t+\Delta t)=E\left\{\gamma_{o} \frac{1}{N} \sum_{n=0}^{N-1}\left|H_{k}(m N+n, t)\right|^{2}\right\} \\
& \approx \gamma_{o}\left|R_{k}(\Delta t)\right|^{2} E\left\{\left|\tilde{H}_{k}(m, t)\right|^{2}\right\}+\gamma_{o}\left(1-\left|R_{k}(\Delta t)\right|^{2}\right) .
\end{aligned}
$$

Letting $\pi_{m}$ be the index number of the MS assigned to the $m$-th subchannel as

$$
\pi_{m}=\arg \max _{k=0, \ldots, K-1}\left\{\gamma_{k}(m, t)\right\}
$$




$$
\gamma_{o} E\left\{\left|\tilde{H}_{\pi_{m}}(m, t)\right|^{2}\right\} \geq \gamma_{o}
$$

This is mainly due to that each MS transmits the probing signal over subchannels having high SNR. Letting $\Gamma_{\pi_{m}} \cong \gamma_{o} E\left\{\left|\tilde{H}_{\pi_{m}}(m, t)\right|^{2}\right\}$, the expected SNR of the $m$-th subchannel at time $t+\Delta t$ can be represented as

$$
\gamma_{\pi_{m}}(m, t+\Delta t)=\left|R_{k}(\Delta t)\right|^{2} \Gamma_{\pi_{m}}+\left(1-\left|R_{k}(\Delta t)\right|^{2}\right) \gamma_{o}
$$

It can be seen that as $\Delta t$ increases, $\left|R_{k}(\Delta t)\right|^{2}$ decreases, yielding the reduction of the capacity.

\section{PERFormance EVALUATION}

The performance of the proposed scheme is verified by computer simulation. The simulation environment is almost identical to [4] and summarized in Table I, where each of 24 subchannels comprises 36 subcarriers. We assume ideal estimation of SNR, and that perfect AMC is implied to clearly examine the effect of probing schemes on the system performance. Threshold $\alpha_{k}$ of main-probing is determined by

$$
\begin{aligned}
\alpha_{k}=(1 & -\beta)\left(\max \left\{\gamma_{k}(m, t)\right\}\right) \\
+ & \beta\left(\min \left\{\gamma_{k}(m, t)\right\}\right), \quad 0 \leq \beta \leq 1
\end{aligned}
$$

where $\beta$ is a system parameter applied to all MSs to determine the number of subchannels for the main-probing. For example, when $\beta=0$, each MS sends the main-probing signal through only one subchannel having the maximum SNR and when $\beta=1$, each MS transmits the main-probing signal over all the subchannels.

Fig. 2 depicts the throughput performance according to the subcarrier interval $d$ in the pre-probing when $K=4$ and $\beta=0.65$. It can be seen that as $d$ increases, the throughput performance decreases since the number of the pre-probing signal per subchannel is reduced, yielding a large channel estimation error. And the performance rapidly deteriorates as $d$ increases in highly frequency selective environments (i.e., large delay spread) due to inaccurate channel information.

Fig. 3 depicts the throughput performance of the proposed scheme according to $\beta$ (or equivalently the number of subchannels for the main-probing) when $K=36$ and $d=36$ (i.e., only one subcarrier per subchannel). For comparison, we consider the use of full channel information for the scheduling. It can be seen that as $\beta$ increases, the performance of the proposed scheme rapidly increases. This is mainly due to the fact that as the number of subchannels for the main-probing increases, each subchannel can be probed at least by one MS.

Fig. 4 depicts the throughput performance of the proposed

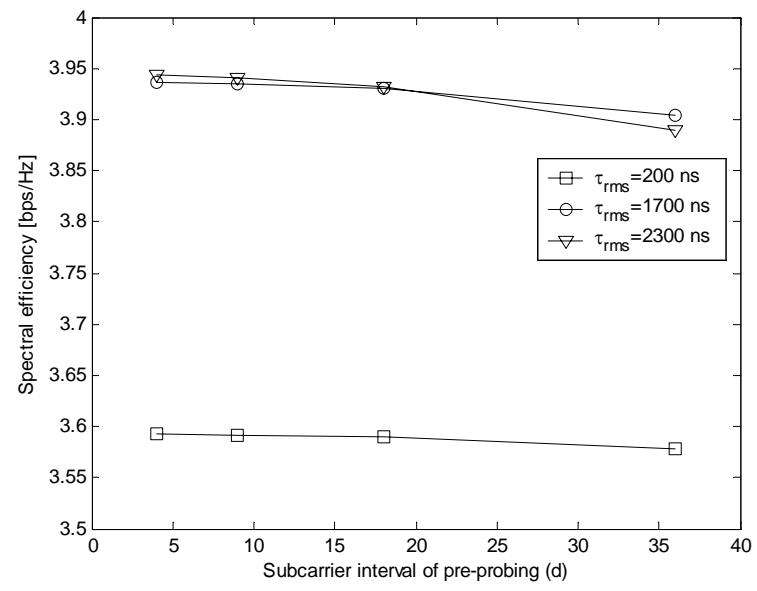

Fig. 2. Throughput performance according to $d$.

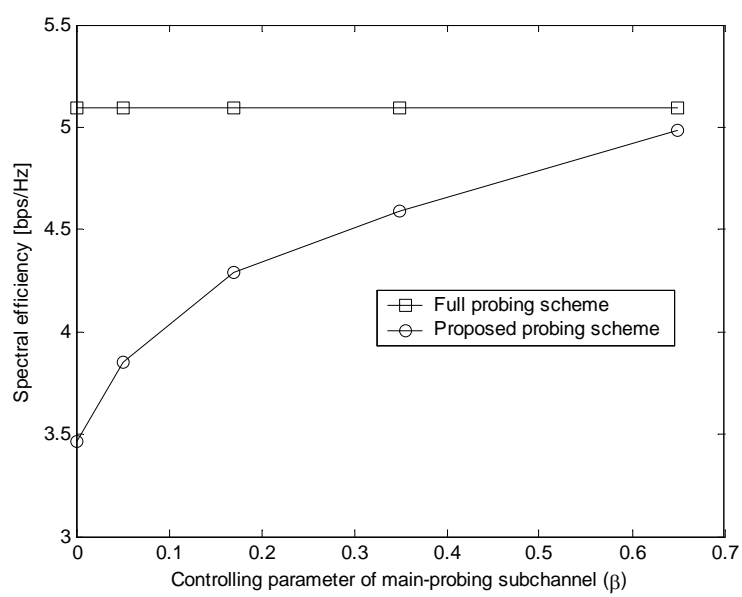

Fig. 3. Throughput performance according to $\beta$.

scheme according to the pre-probing interval $T_{1}$ when $K=4$, $d=4$ and $\beta=0$. It can be seen that as $T_{1}$ increases, the throughput performance deteriorates. This is mainly due to the fact that as the larger $T_{1}$, the higher the probability that the channel condition of the selected subchannels is not good. It can also be seen that as the Doppler spread (or the user mobility) increases, the performance is reduced since the time correlation of the channel decreases.

Fig. 5 depicts the throughput performance of the proposed scheme according to the main-probing interval $T_{2}$ when $K=4, d=4$ and $\beta=0$. It can be seen that as $T_{2}$ increases, the system performance deteriorates. This is mainly due to the fact that data rate determined by the man-probing process is no longer optimum to the current channel condition. Besides, as the Doppler spread increases, the performance loss increases since the channel correlation in time decreases. 


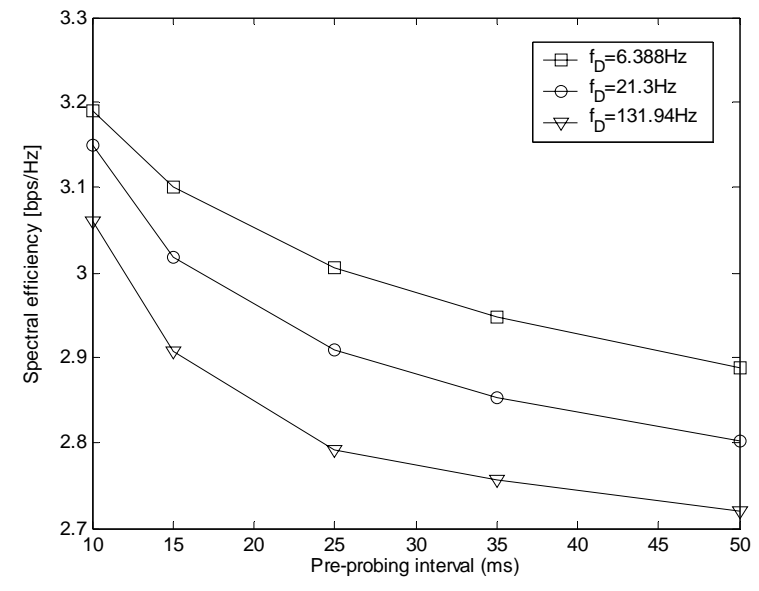

Fig. 4. Throughput performance according to $T_{1}$.

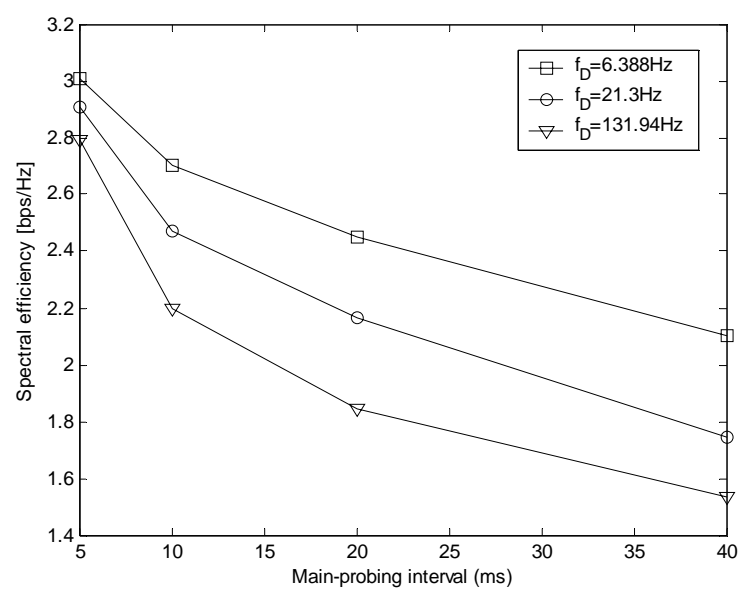

Fig. 5. Throughput performance according to $T_{2}$.

Fig. 6 depicts the throughput performance of the proposed scheme according to the number of MSs when $d=36$ and $\beta=0$. When $K=36$, it can be seen that when the same amount of probing signal is used, the proposed scheme improves the throughput performance by about $13 \%$ compared to the use of conventional IEEE 802.16e scheme that determines the probing subchannels without considering the channel state of MS. It can also be seen that as the number of MSs increases, the proposed scheme can provide the throughput performance comparable to the full probing scheme while significantly reducing the signaling burden for the probing.

\section{CONCLUSIONS}

In this paper, we have proposed a new channel probing scheme in the uplink of an OFDM-based multi-user wireless system. By first determining good subchannels and then sending the probing signal over only subchannels in good

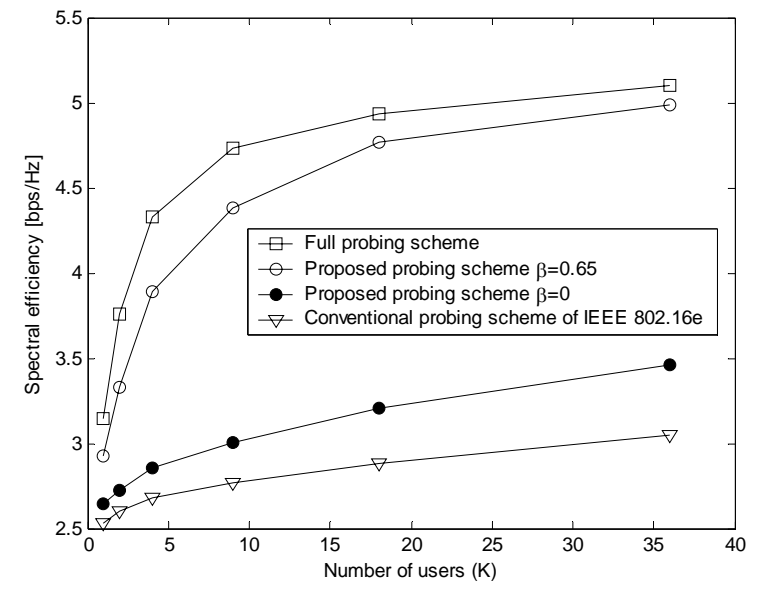

Fig. 6. Throughput performance according to $K$.

condition, the proposed scheme can remarkably reduce the signaling burden for the probing without noticeable performance degradation. It has been shown that the proposed probing scheme provides almost the same throughput as the full probing scheme as the number of MSs increases. The simulation results show that the proposed scheme outperforms the conventional scheme and can remarkably reduce the signaling burden for the probing compared to the full probing scheme.

\section{REFERENCES}

[1] R. W. Heath Jr., M. Airy and A. J. Paulaj, "Multiuser diversity for MIMO wireless systems with linear receivers,” in Proc. Asilomar Conf. Signals, System and Computers, vol. 2, pp. 1194-1199, Nov. 2001.

[2] W. Rhee, W. Yu and J. M. Cioffi, "Utilizing multiuser diversity for multiple antenna systems," in Proc. Wireless Commun. Network Conf., vol. 1, pp. 420-425, Sept. 2000.

[3] T. A. Thomas, K. L. Baum and P. Sartori, "Obtaining channel knowledge for closed-loop multi-stream broadband MIMO-OFDM communications using direct channel feedback," in Proc. Globecom'05 fall., vol. 6, pp. 5, Nov. 2005.

[4] IEEE P802.16e, Draft IEEE standard for local and metropolitan area networks, Sept. 2004.

[5] G. Auer, A. Dammann and S. Sand, "Channel estimation for OFDM systems with multiple transmit antennas by exploiting the properties of the discrete Fourier transform,” in Proc. PIMRC'03, vol. 2, pp 19541958, Sept. 2003

[6] C. S. Suh and C. S. Hwang, "Channel estimation technique for mitigating ICI in MIMO-OFDM cellular systems,” in Proc. Globecom'04 fall., vol. 4, pp 2679-2683, Nov. 2004.

[7] S. Catreux, et.al., "Adaptive modulation and MIMO coding for broadband wireless data networks," IEEE Commun. Mag., vol. 40, pp. 108-115, June 2002.

[8] M. Gong, L. Qiu and I. Zhu, "An efficiency multiuser diversity scheme with partial feedback on common uplink channel," in Proc. VTC'05 spring., vol. 3, pp. 1894-1897, May 2005.

[9] J. G. Proakis, Digital communications, McGraw-Hill, 4-th ed., 2001.

[10] W. C. Jakes, Microwave mobile communication, $2^{\text {nd }}$ ed. Piscataway, NJ: IEEE Press, 1994.

[11] M. Alouini and A. J. Goldsmith, "Capacity of Rayleigh fading channels under different adaptive transmission and diversity-combining Techniques,” IEEE Commun. Mag., vol. 48, pp. 1165-1181, July 1999. 